\title{
Carnets
}

Revue électronique d'études françaises de l'APEF

Première Série - 1 | 2009

La mer... dans tous ses états

\section{La mer vagabonde du jeune commandeur de Prévost}

Ana Alexandra Seabra De Carvalho

\section{OpenEdition}

1 Journals

\section{Édition électronique}

URL : http://journals.openedition.org/carnets/2890

DOI : 10.4000/carnets.2890

ISSN : 1646-7698

Éditeur

APEF

Édition imprimée

Pagination : 69-80

\section{Référence électronique}

Ana Alexandra Seabra De Carvalho, "La mer vagabonde du jeune commandeur de Prévost », Carnets

[En ligne], Première Série - 1 | 2009, mis en ligne le 13 juin 2018, consulté le 01 mai 2019. URL : http:// journals.openedition.org/carnets/2890 ; DOI : 10.4000/carnets.2890

\section{(c) (1) (8)}

Carnets est mis à disposition selon les termes de la licence Creative Commons - Atribution - Pas d'utilisation commerciale 4.0 International. 


\title{
LA MER VAGABONDE DU JEUNE COMMANDEUR DE PREVOST
}

\author{
Ana Alexandra Seabra de Carvalho \\ Universidade do Algarve \\ aacarva@ualg.pt
}

\section{Résumé}

Nous proposons ici une lecture centrée sur la thématique de la mer à propos d'un roman moins connu de l'Abbé Prévost intitulé Mémoires pour servir à l'Histoire de Malte, ou Histoire de la jeunesse du Commandeur (1741). Dans ce roman d'aventures barbaresques, la mer constitue le cadre spatial de l'aventure et de l'amour, aussi bien que le lieu de la réflexion, de la mésaventure, de la souffrance et du combat à la fois guerrier et intérieur du héros-narrateur, un chevalier de Malte qui tombe amoureux d'une belle jeune fille, pourtant indigne de lui. On peut y observer le déploiement de la thématique de la dérive du moi et de la dissolution du sujet, aussi bien que les orages de l'errance et de la passion masqués d'un romanesque effervescent. Mais à la fin le jeune commandeur se sépare de sa maîtresse, devenue défigurée par la petite vérole, ce qui souligne la conception pessimiste prévostienne de l'amour maudit.

\begin{abstract}
In this paper we propose an analysis emphasizing the theme of the Sea at presence in one of the less known of Prévost's novels entitled Mémoires pour servir à l'Histoire de Malte, ou Histoire de la jeunesse du Commandeur (1741). In this barbaric adventure novel, the sea constitutes, on one hand, the spatial frame for adventure and love and, on the other, the place for reflection, misfortune, suffering, sea battles, as well as for the narrating hero's inner struggle, a Malta's chevalier who falls in love with a beautiful young girl, however unworthy of him. Here we can verify the development of themes such as the drift and dissolution of the self, the storms of wandering and love masked by an effervescent Romanesque. In the end, however, the young commendator leaves his mistress, disfigured by smallpox, which emphasizes Prévost's pessimistic conception of fatal love.
\end{abstract}

Mots-clés: Prévost, Roman d'aventures, Mer, Amour, Vagabondage

Keywords: Prévost, Adventure novel, Sea, Love, Wandering 
L'enfance n'est sinon qu'une sterile fleur,

La jeunesse, qu'ardeur d'une fumiere vaine,

Virilité qu'ennuy, que labeur, et que peine,

Viellesse que chagrin, repentance, et douleur,

Nos jeus que desplaisirs, nos bon-heurs que mal-heur, Nos thresors et nos biens, que tourment, et que geine, Nos libertez que laqs, que prisons, et que chaine, Nostre aise, que mal-aise et nostre ris que pleur;

Passer d'un âge à l'autre, est s'en aller au change D'un bien plus petit mal, en un mal plus estrange

Qui nous pousse en un lieu d'où personne ne sort.

Nostre vie est semblable à la mer vagabonde, Où le flot suit le flot, et l'onde pousse l'onde,

Surgissant à la fin au havre de la mort.

Jean-Baptiste Chassignet, Le Mespris de la Vie et Consolation contre la Mort (1594), sonnet 53

Nous proposons ici une lecture centrée sur la thématique de la mer à propos d'un roman moins connu de l'Abbé Prévost intitulé Mémoires pour servir à l'Histoire de Malte, ou Histoire de la jeunesse du Commandeur, publié en deux tomes à Amsterdam en 1741 (Prévost, 1996). Roman d'aventures maritimes à tiroirs, divisé en deux parties de longueur à peu près égale, il développe la thématique de la mer à côté de celles de la dérive du moi et de la dissolution du sujet, comme l'a très bien remarqué René Démoris (Démoris, 1996: 21), où les orages de l'errance et de la passion se masquent d'un romanesque effervescent (Fabre, 1979: 104) dans un récit d'aventures barbaresques. La succession rapide et enchevêtrée des événements jointe à la lutte du héros contre les caprices de l'amour et du hasard dévoilent la marque du romancier. Aussi les récits y occupent-ils une place prépondérante par rapport à la brièveté des dialogues. Les descriptions se réduisent à des indications sommaires et les réflexions aussi bien que les remarques psychologiques sont brèves et disséminées tout au long du texte, comme il convient au genre aventurier; ce sont d'ailleurs des caractéristiques qui se trouvent, en général, présentes dans toute l'œuvre romanesque de Prévost.

Dans ce roman, la mer fonctionne surtout comme le cadre spatial de l'aventure et de l'amour, mais elle est aussi le lieu de la réflexion, de la mésaventure, de la souffrance et du combat à la fois guerrier et intérieur du héros-narrateur. Celui-ci est un chevalier de Malte qui tombe amoureux d'une jeune fille indigne de lui, symboliquement appelée Helena, la séduit 
et passe avec elle quelques mois à Naples. Pourtant, il ne veut pas sacrifier sa foi et sa vertu à l'amour et choisit de faire carrière à l'ordre de Malte. Mais quand sa douce et fidèle bienaimée est enlevée par les corsaires, il décide d'aller la chercher au Maroc aux dépens de sa carrière de commandeur. Cependant, le destin frappe à nouveau et il ne la reconnaît même pas quand il la retrouve toute défigurée par la petite vérole, ce qui refroidit sa passion pour cette fille devenue si hideuse ${ }^{1}$. À la fin, le héros se sépare définitivement de sa maîtresse, car il refuse un attachement qu'il croit le conduire à l'abîme, dénouement qui souligne la conception pessimiste prévostienne de l'amour maudit.

Dans ces mémoires, le romancier donne la parole à un héros-narrateur "lucide, mais triste et froid", selon Henri Coulet, qui balance beaucoup avant sa décision finale, car "le passé n'est plus rien dans sa vie, il est devenu tout entier l'homme de son Ordre, et son récit est l'un des plus désenchantés et l'un des plus impudiques que Prévost ait écrits" (Coulet, 1967: 363). En effet, le chevalier hésite entre son désir de l'ordre et le désordre de son désir amoureux, entraîné par des contradictions entre le présent et le passé, ses propres désirs et aspirations et ceux de sa maîtresse. Cependant, il y a aussi la dérive herméneutique et l'incapacité à saisir la réalité au-delà des mots et des interprétations, muables comme l'onde vagabonde.

Une fois encore, Prévost se montre, dans ce bref roman d'aventures amoureuses et maritimes, comme un romancier accompli "par son art de développer des intrigues complexes et par sa connaissance de l'âme humaine, par son sens de la réalité" (Lemaître, 1994: 673). Cependant, comme le remarque Henri Coulet, dans le réalisme de Prévost, "le détail concret n'est jamais présenté pour lui-même, mais toujours de façon symbolique et sentimentale" (Coulet, 1967: 358). II en va de même pour la description de la mer dans tous ses états, car ici la mer et l'amour vont de pair. Prévost romancier pose toujours des questions sur le bonheur et les passions humaines, il "exprime les angoisses et les souffrances de héros victimes du destin, dont la faiblesse est propre à émouvoir le lecteur" (Lemaître, 1994: 673). II s'agit, en effet, selon Henri Coulet, de personnages exceptionnels: "tous se considèrent comme particulièrement désignés par le destin ou la providence pour des aventures et des malheurs qui les distinguent des autres hommes" (Coulet, 1967: 358). Ils se caractérisent par "un superlatif de mauvaise fortune et de sensibilité: ces [...] termes vont ensemble, des âmes d'élite ne pouvant se révéler que dans des épreuves inouïes. L'invraisemblance est donc le climat naturel des romans de Prévost" (Coulet, 1967: 359), et ce sont des œuvres qui mêlent réalisme et romanesque. Le lecteur de La jeunesse du commandeur, par exemple, sera surtout sensible "à la séduction de ce jeune fou qui abandonne le patrimoine pour faire de la mer [...] le théâtre de ses exploits, comme les

\footnotetext{
${ }^{1}$ Cf. Prévost, 1996: 297.
} 
héros de Gomberville et de La Calprenède" (Démoris, 1996: 8). L'amateur de récits d'aventures maritimes sera comblé par la profusion d'événements ravissants, car "ici les naufrages ne sont pas métaphoriques: tempêtes, orages, pirates et abordages sont bien réels et c'est à une 'mer impétueuse' que le héros doit de relâcher à Orbitello, et de voir, pour la première fois, cette Helena qu'il va aimer" (ibid.). On aura encore des "retrouvailles au hasard d'un abordage, sauvetage d'un noble espagnol arraché aux eaux qui devient l'ami fidèle, vagabondages passionnées à travers toute la Méditerranée à la quête de l'objet aimé" (ibid.).

Pourtant, ce roman maritime est encore à la fois un roman psychologique et une réflexion sur l'écriture romanesque, capable de plaire, malgré tout, au lecteur le plus exigeant de Prévost, comme le remarque aussi René Démoris:

Et puis cela dérive, ce terme de navigation n'étant pas impropre, peut-être, dans un roman aussi exemplairement maritime, à rendre compte à la fois du destin du héros narrateur et de la démarche du romancier dévoilant, par touches insensibles, le leurre de la perspective héroïque. Un beau fruit, pour le dire autrement, dont on découvrirait, non sans déplaisir, qu'il est gâté. II se peut [...] que Prévost ait écrit trop vite. Mais la manière dont est menée cette opération déceptive relève du très grand art. On peut tenir pour placages les histoires romanesques [...] de Perés le noble espagnol et de Junius le commandeur renégat et aventurier mégalomane [...], mais ces aventures ne sont-elles pas là pour marquer aussi la déliquescence de l'histoire principale, la manière dont, à l'image du héros, elle se laisse entraîner hors de son propos? (Démoris, 1996: 9)

Cependant, le choix de la forme romanesque traditionnelle permet à Prévost d'aller plus loin. C'est que le cliché bien connu de son lecteur avide d'aventures n'est pas là l'essentiel mais le moyen de le porter au-delà de cette surface divertissante et de le faire plonger au gouffre amer des conflits des passions humaines et de la crise sentimentale. En fait, victime de la crise sentimentale, "le commandeur de Malte rompt avec sa maîtresse, devenue laide et insupportable, mais ce n'est pas sans difficultés ni déchirement" (Sgard, 1975: 361). Le pessimisme présent dans toute l'œuvre de Prévost se fonde, selon Jean Sgard, "sur l'échec de l'amour" et alors "la faillite de l'amour ouvre les portes au désespoir, au néant" (Sgard, 1975: 362). Ce commandeur de Malte est "un aventurier sans foi ni loi: courir les mers, piller, se créer un royaume pour lui seul, vivre pour l'amour en un jardin et abandonner sa maîtresse quand elle vieillit, tels sont ses faits d'armes" (ibid.).

Les mots de Henri Coulet que nous venons de citer ci-dessus reprennent la question de notre début. II s'agit bien d'un roman d'aventures à la fois amoureuses et guerrières dans le goût du genre héroïque baroque, où la mer Méditerranée, la voie de communication par 
excellence dans le texte, est le théâtre des exploits du héros et de ses compagnons qui y vagabondent à plaisir, tout en souffrant les tempêtes, les orages, les abordages, les pillages et les enlèvements des corsaires turcs, les naufrages, etc. Roman maritime, donc, pour la thématique. Pourtant, il dérive, comme l'a remarqué René Démoris cité plus haut, en se métamorphosant en un roman de la dissolution du sujet, mais qui remet aussi en cause les lois du romanesque héroïque. En outre, cette mer tumultueuse est l'agent du destin, en procurant les rencontres essentielles du récit (l'amante et l'ami fidèle du héros); tandis que le temps de la navigation par une mer calme sert surtout à la méditation et à la réflexion, à la séduction et à l'amour.

Cependant, quelque chose a changé et ce chevalier de Malte est loin d'être un saint. En effet, ses faits d'armes ne correspondent plus à l'héroïsme des exploits de ses devanciers baroques pour propager la foi. Au contraire, il désire plutôt vivre des aventures semblables à celles des héros de ses lectures romanesques:

je reçus la croix [de Malte] presque en naissant; mais la mort de mon aîné m'ayant fait succéder à tous ses droits, on fut surpris qu'à l'âge de dix-huit ans, et lorsque tout semblait m'appeler aux fonctions du chef d'une grosse maison, je parlai de me rendre à Malte pour mes caravanes, et d'abandonner à mes cadets toutes mes prétentions. J'avais pris ce goût dans la lecture. Rien ne m'avait paru si noble et si grand que ma première vocation, et je ne pus me persuader que des avantages aussi frivoles que les biens de la fortune dussent balancer un sentiment qui me paraissait fondé sur l'honneur et la raison (Prévost, 1996: 30).

Malgré les résistances de sa famille, il est donc parti avec deux voisins qui entreprenaient le même voyage, tout d'abord par une mer calme mais qui va bientôt devenir orageuse: "et notre navigation fut heureuse jusqu'à l'entrée de la mer de Gênes; mais un vent impétueux nous ayant forcés de ranger la côte, le capitaine prit le parti de relâcher pour quelques jours dans le port d'Orbitello" (op. cit: 30; nous soulignons). La toute première référence à la mer dans le roman est déjà à la fois réaliste, romanesque et symbolique, car ce "vent impétueux", porteur d'une tempête qui force le capitaine à chercher l'abri du port d'Orbitello pour y réparer son vaisseau, agit par la main du destin, puisque c'est là que, tout de suite, le héros rencontrera pour la première fois la belle jeune fille, Helena, dont il deviendra éperdument amoureux par la suite (op. cit:: 32-33).

Quelque temps après on quitte le rivage d'Orbitello pour entreprendre à nouveau le voyage maritime vers Malte, cette fois-ci sans incidents. Après un séjour de quinze jours dans cette île, le héros-narrateur doit à nouveau se remettre en mer. Une fois encore, les conditions de navigation semblent au début être les meilleures: "Nous nous mîmes en mer 
dans un temps qui nous promettait la plus heureuse navigation" (op. cit:: 33). Pourtant, le destin frappe à nouveau par le moyen d'une violente tempête: "mais par le même sort qui m'a toujours rendu cet élément funeste, à peine fûmes-nous éloignés de la côte qu'une affreuse tempête sépara notre vaisseau des deux autres" (ibid.). Alors, le navire devient le jouet du vent et des flots et l'équipage va se retrouver de l'autre côté de la Méditerranée, en territoire ennemi: "Nous fûmes jetés vers la côte d'Afrique, où dans le triste état de notre manœuvre nous ne vîmes rien de plus favorable que de nous mettre à l'abri dans quelque rade. II fallait la choisir écartée" (op. cit:: 33-34). Les lois du récit romanesque obligent, et le lecteur doit donc espérer quelque événement saisissant. Le voilà:

Mais comme nous approchions d'une baie déserte, où deux montagnes nous paraissaient propres à nous mettre à couvert, nous fûmes surpris d'entendre des cris perçants dans un lieu qui n'était point habité: le temps, qui était fort épais, ne nous permettait point de découvrir ceux qui nous avaient aperçus; c'était quatre misérables qui luttaient contre les flots, sur un mât qu'ils tenaient embrassé, et dont le sort nous apprit que le nôtre pouvait devenir encore plus malheureux. Quelque ardeur que la seule humanité nous donnât pour les secourir, nous étions encore si agités par le mouvement des vagues, qu'il ne fut aise ni à eux de s'approcher de nous, ni à la chaloupe de s'avancer jusqu'à eux. Cependant deux de ces infortunés perdirent enfin la respiration et les forces. Ils lâchèrent le mât, et nous eûmes la douleur de les voir périr à nos yeux. J'excitais par mes cris et par l'offre d'une grosse récompense, les matelots qui s'étaient mis dans la chaloupe; mais tous leurs efforts ne pouvant les faire approcher du mât, nous vîmes périr encore un des malheureux, à qui nous voulions donner du secours (op. cit.: 34-35).

Cette scène touchante d'un sauvetage presque manqué (puisque, au risque d'être lui-même englouti par les flots, le héros-narrateur arrive quand même à sauver le quatrième naufragé, Perés, un noble espagnol disgracié qui deviendra son ami et mentor) correspond à la deuxième référence à la mer dans le roman.

Il s'agit à nouveau d'une mer tranquille au début de la scène, mais qui tout d'un coup devient terriblement tempétueuse et cause du naufrage. Ensuite, comme par miracle, elle va s'apaiser à la fin de la scène. Tout comme la première fois, son véritable intérêt est d'introduire un des personnages fondamentaux du roman, ici l'ami Perés:

je me jetai dans la mer, pour faire avec la main ce qui m'avait si mal réussi avec le croc. Cette folle générosité devait rendre ma perte certaine. Je me trouvais tout d'un coup dans un péril beaucoup plus grand que le malheureux même que je voulais secourir; mais par un miracle dont toute ma reconnaissance ne m'acquittera jamais envers le ciel, le vent qui avait soufflé si impétueusement jusqu'alors, perdit en un 
moment toute sa violence, et le mouvement même des vagues diminua sensiblement (op. cit.: 35).

Sauvé à son tour par les matelots dès que la tempête se fut calmée, le hérosnarrateur reste pendant plus de deux heures sans connaissance. Ensuite, il prie l'étranger de lui raconter son histoire et les circonstances de son naufrage (op. cit: $37-67$ ). On a là une double perspective de cette calamité maritime, ce qui renforce l'intérêt romanesque du lecteur amateur du genre, d'un côté, et constitue, de l'autre, une première esquisse du caractère du noble Perés, de sa présence d'esprit et de son courage:

Vous savez à quelle heure commença la tempête, puisque vous l'avez essuyée. II fut bien moins question de penser à la fuite qu'à la conservation du vaisseau que nous voulions abandonner. Je me prêtai au travail comme le moindre matelot; ce qui n'empêcha point que dans l'affreux désordre où était l'équipage, je ne visse l'Espagnol et ses deux compagnons détacher la chaloupe [...]. Je le perdis de vue dans l'obscurité, et ne pensant moi-même qu'à défendre ma vie, à l'instant d'un naufrage inévitable, je me saisis d'un mât fracassé, que je jetai assez adroitement en mer pour m'élancer dessus au même moment. Le domestique de Dom Antonio [...] se jeta après moi avec le même bonheur. Notre exemple anima quelques autres matelots à nous imiter. Ils n'ont pas résisté sans doute à l'impétuosité des flots, puisque cette voie de salut n'a été favorable que pour moi; mais j'errais depuis plus de six heures au gré du vent, et je voyais encore quelques-uns de mes compagnons attachés au mât. Les cris que la vue de votre vaisseau nous a fait pousser comme de concert ont attiré vos yeux sur nous; mais lorsque mon agitation redoublait par des espérances si prochaines, j'ai senti à la légèreté du mât que mes malheureux compagnons périssaient successivement. J'ai tiré de nouvelles forces du malheur d'autrui. L'ardeur que j'ai remarquée à vos gens pour me secourir m'a fait même éprouver que dans l'extrémité du péril on peut être sensible à la joie. Mais que dis-je? Je l'ai été jusqu'au transport, à la compassion et à la reconnaissance, lorsqu'après vous avoir vu faire mille efforts pour vous saisir de mon mât, je me suis aperçu, en vous voyant disparaître, que vous aviez été englouti par les flots, et j'aurais abandonné mille fois l'instrument de mon salut, si j'avais eu la moindre espérance de racheter votre vie aux dépens de la mienne. Enfin j'ai été enlevé, au milieu de cette agitation, par des mains puissantes, qui m'ont couché dans la chaloupe. Mon premier mouvement a été de presser mes libérateurs de vous rendre le même office. Ils vous cherchaient, et la violence de la tempête étant extrêmement diminuée, ils n'ont pas eu de peine à vous trouver (op. cit.: 65-66; nous soulignons). 
Leur retour à Malte après la terrible tempête est regardé comme un vrai miracle. Perés et le chevalier deviennent des amis inséparables et ce dernier, avec l'argent de sa large pension et les connaissances nautiques de l'Espagnol, s'achète "un des meilleurs voiliers que les Turcs eussent dans [la] mer [de Venise]" et un équipage de soixante hommes bien choisis et résolus, cinquante soldats et dix matelots, "capables de toutes sortes d'entreprises" (op. cit.: 73). Le vaisseau est nommé les deux Commandants, car il n'y aura point de distinction entre les deux commandants, le Français et l'Espagnol. Ainsi équipés, leur projet est de retourner à l'île de Malte et de se placer sous les ordres du grand maître. Pourtant, romanesque oblige!, le destin est aux aguets, comme le remarque le narrateur des Mémoires:

La défiance de notre soumission, que j'avais cru remarquer au grand maître, nous fît prendre le parti de retourner d'abord à Malte, pour recevoir les premiers ordres à la tête de nos gens; mais la fortune, qui nous destinait plus de gloire que de bonheur et de richesses, nous préparait sur la route une rencontre dont toutes les aventures de ma jeunesse ont pris leur source (op. cit:: 73-74; nous soulignons).

Cette rencontre fatale est celle d'Helena, retrouvée enlevée avec sa mère dans le premier vaisseau turc abordé furieusement et victorieusement par le héros-narrateur et tout son équipage à peine remis en mer:

La première vue de notre proie nous promit peu de richesses; et nous apprîmes au même moment que nous n'avions affaire qu'à des pirates de Dulcigno, qui n'avaient rien à risquer que leur vie et leur vaisseau. Cependant aussitôt que nous les eûmes fait enchaîner, il se présenta plusieurs femmes, qui vinrent nous remercier comme leurs libérateurs. Elles nous racontèrent que s'étant embarquées sur la côte de Gênes pour se rendre à Malte, elles avaient eu le malheur d'être arrêtées par ces corsaires [...]. Tout étant si tranquille autour de nous qu'il ne nous restait qu'à voguer tranquillement vers Malte, nous cherchâmes à nous amuser dans la compagnie de ceux qui nous devaient leur liberté. Nous ne prévoyions ni l'un ni l'autre que nous y allions trouver la perte de la nôtre, et l'origine d'autant d'infortunes que de plaisirs. Ces deux dames, dont je ne me remis pas tout d'un coup le visage, étaient la maîtresse et la fille du commandeur de M., qui nous avait traités avec tant de politesse dans le voisinage d'Orbitello. Le commandeur étant mort, elles avaient pris aussitôt le parti de retourner à Malte, et les corsaires les avaient enlevées dans leur route (op. cit.: 75-76).

Alors, le chevalier éprouve à la fois, tout en voguant tranquillement en pleine mer, toute la violence du coup de foudre amoureux et l'aiguillon de la jalousie: 
je lui trouvai plus de charmes qu'une femme n'en a jamais réunis. Ce fut l'impression d'un seul moment, et l'effet en devint tout d'un coup si terrible, que ne pensant pas même à m'en défendre, je m'approchai d'elle avec une avide impatience, comme si tout mon bonheur eût déjà consisté à la voir de près, à la contempler, et à ne plus m'éloigner d'elle un moment.

Mais la force d'un sentiment si peu réfléchi me fit découvrir avec la même promptitude que j'avais un rival dans mon ami, et que le cœur de Perés éprouvait tout ce qui se passait dans le mien. J'évite également de retracer ici l'excès de mon plaisir et de ma peine. Peut-être suis-je le seul exemple d'un amour né au milieu de tant de douleur (op. cit: : 76-77).

C'est aussi à bord de son vaisseau que, sous le prétexte d'une fête, le chevalier enlève Helena, sa mère et une Espagnole: "nous commençâmes par un grand dîner qui fut poussé jusqu'à la nuit, et les ténèbres n'eurent pas plus tôt commencé à s'épaissir que Perés donna ordre secrètement qu'on mît à la voile" (op. cit:: 91). On assiste, alors, aux déclarations amoureuses des deux amis faites en pleine mer. Mais, encore une fois, la fatalité est aux aguets:

Perés, peu touché par l'amour, ne trouvait qu'un sujet de raillerie dans la ressemblance de nos aventures. Je prévois notre sort, ajouta-t-il. Après avoir regardé nos dames comme l'agrément de notre route, peut-être en vont-elles faire le supplice; et nous serons fort heureux à la fin de trouver quelque moyen de nous en défaire honnêtement.

[...] Ce qui vous paraît un badinage est la plus sérieuse affaire de ma vie. J'aime plus que jamais depuis que je suis sûr d'être aimé. Ma passion est devenue si nécessaire à ma vie, que je préfèrerais la mort à la nécessité de me séparer d'Helena. Je la verrai malgré sa mère, je ferai mon bonheur de sa tendresse, je la rendrai heureuse ellemême par l'ardeur et la constance de mes sentiments (op. cit.: 102-103; nous soulignons).

Or, ces derniers mots du chevalier seront démentis par la suite, après la terrible métamorphose de sa maîtresse et malgré sa désobéissance hardie au grand maître, en entreprenant des "vagabondages passionnés à travers toute la Méditerranée à la quête de l'objet aimé" (Démoris, 1996: 8)²:

\footnotetext{
${ }^{2}$ Cf. Prévost, 1996: 200-262.
} 
J'étais plein d'elle, je ne parlais que de ses charmes et des transports que j'allais ressentir en la voyant. Je priais le ciel d'anéantir les heures qui retardaient son arrivée. Elle parut enfin, ou plutôt apprenant qu'elle montait l'escalier, je commençais à me précipiter vers la porte, lorsque Perés l'ouvrit, et me présenta une jeune personne qui fut absolument inconnue pour moi. Je demeurai interdit, en cherchant à quoi cette plaisanterie pouvait aboutir. Je voyais une fille de la taille et de l'âge d'Helena; mais j'avais vu peu de visages qui m'eussent paru aussi désagréables. Une peau difforme; les yeux louches, une blancheur fade et dégoûtante. En fixant néanmoins mes regards sur ce fantôme, je ne laissais pas d'y démêler quelque chose qui ne m'était point étranger. J'allais marquer mon étonnement à Perés, et lui demander pourquoi je le voyais sans Helena; mais il prévint ma question et mes plaintes: Je vous la rends, me dit-il, et vous n'avez pas eu plus d'empressement qu'elle pour cet heureux moment. Sa maladie l'a un peu défigurée, ajouta-t-il, et je ne l'ai pas reconnue tout d'un coup. Mais l'amour pénètre au travers de tous les voiles, et vous reconnaissez sans doute votre chère Helena. [...] Pour moi, qui n'aurais pas été plus abattu d'un coup de foudre, je demeurai quelques moments à considérer l'objet de ma tendresse, et je cherchais dans ses traits quelques restes de l'image que j'en conservais encore au fond du cœur. Un froid inexprimable, qui me glaçait le sang à mesure que cette nouvelle figure semblait effacer l'autre, m'empêcha d'ouvrir les bras pour l'embrasser. Cependant la bienséance me porta enfin à lui faire quelques caresses. Je m'assis près d'elle; je tâchai de rappeler le souvenir de mes anciens sentiments, pour suppléer à ceux que mon cœur me refusait (op. cit.: 297-298; nous soulignons).

Après ce coup de foudre à l'envers, le chevalier s'efforcera vainement de revenir sur ses premiers sentiments, mais à la fin ce sera le triomphe du dégoût. Alors, il se forme un plan pour s'en débarrasser, en l'offrant en mariage à son valet (op. cit. 322). Dépitée, Helena refuse cette proposition et mène le chevalier à l'engager au service de sa maison. Quelque temps après, elle le séduit et il se livre avec elle aux dernières faiblesses (op. cit. 371).

Pourtant, même dans les bras de l'amour, le chevalier est rappelé encore une fois à ses services, cette fois-ci comme l'ambassadeur de la religion chrétienne, ce qui entraîne la décision de se défaire de son vaisseau, c'est-à-dire, d'abandonner sa carrière maritime aussi bien que sa maîtresse:

Ainsi tous mes sentiments allaient reprendre leur cours, et mon imagination m'aurait représenté à la fin une maîtresse plus aimable que jamais, lorsque le souvenir de mes services me fit choisir par le grand maître pour ambassadeur de la religion à la cour de... C'était m'ouvrir une nouvelle carrière, où j'entrais d'autant plus volontiers que 
ces longues agitations commençaient à me faire souhaiter le repos. Je pris aussitôt la résolution de me défaire de mon vaisseau (op. cit.: 371-372; nous soulignons).

Mais Helena, en s'apercevant de cette nouvelle disposition, essaye de le détourner de son projet. En ouvrant les yeux sur les nouvelles chaînes dont il s'était chargé et en sentant son cœur beaucoup plus engagé qu'il ne le pensait, le chevalier frémit d'un obstacle qu'il s'était formé volontairement. La honte et la raison fortifient sa décision de quitter sa maîtresse défigurée, laquelle accepte de s'enfermer dans un couvent (op. cit:: 373-375). Les derniers mots du roman confirment ces décisions finales, tout en ajoutant ironiquement "les applaudissements du public" envers une conduite aussi "noble" de la part du jeune chevalier:

Je fis quelques efforts pour l'arrêter, non que je condamnasse son dessein, mais dans la seule vue de prendre toutes les mesures qui pouvaient rendre son sort agréable dans la retraite. Et je cessai même de m'opposer à son départ, lorsque j'eus fait réflexion que cette vivacité ne changerait rien à mes soins. Je communiquai mon projet à sa mère, qui ne balança point à l'approuver; et je l'exécutai avec assez de noblesse pour m'attirer les applaudissements du public (op. cit:: 373-5; nous soulignons).

Ici finissent les Mémoires de la jeunesse du commandeur, au moment où il abandonne pour de bon et sa maîtresse et son vaisseau, c'est-à-dire, son vagabondage maritime et ses aventures à la fois amoureuses et avec les corsaires turcs, pour entrer dans la carrière diplomatique de la religion chrétienne sous les auspices du grand maître de l'Ordre de Malte et avec les applaudissements du public. L'orage passionnel, guerrier et maritime s'apaise vite par le dégoût de l'objet aimé et devient désormais tranquille par le choix raisonné d'une vie réglée et pragmatique. 


\section{Bibliographie}

Cahiers Prévost d'Exiles (1984-1993). 10 vols. Grenoble: P.U. Grenoble.

L'Abbé Prévost (1965). Actes du colloque d'Aix-en-Provence (décembre 1963). Aix-en-Provence: Ophrys.

COULET, Henri (1967). "Prévost”. In: Le roman jusqu'à la Révolution. Paris: Armand Colin, pp. $352-$ 364.

DEMORIS, René (1996). "Préface". In: La jeunesse du commandeur (1741). Paris-Genève: Edition René Démoris et Editions Slatkine, pp. 7-21.

FABRE, Jean (1979). "L’Abbé Prévost et la tradition du roman noir". In: Idées sur le roman. De Madame de Lafayette au Marquis de Sade. Paris: Klincksieck, pp. 100-119.

LEMAITRE, Henri (dir.) (1994). Dictionnaire Bordas de littérature française. Paris: Bordas.

MONTY, J.R. (1970). "Les romans de l'Abbé Prévost”. In: Studies on Voltaire, no 78. Genève.

Prevost, Antoine-François (1967a). Histoire du Chevalier Des Grieux et de Manon Lescaut (1731). Edition de Henri Coulet. Paris: Garnier Flammarion.

Prevost, Antoine-François (1967b). La jeunesse du commandeur de ${ }^{* * *}$ (1741). Edition de Henri Coulet. s.I.: Roissard.

Prevost, Antoine-François (1996). La jeunesse du commandeur (1741). Edition de René Démoris. Paris-Genève: Editions Slatkine.

SERMAIN, J.P. (1985). "Rhétorique et roman au XVIII ${ }^{e}$ siècle: l'exemple de Prévost et de Marivaux". In: Studies on Voltaire and the Eighteenth Century, № 233. Oxford: The Voltaire Foundation.

SGARD, Jean (1968). Prévost romancier. Paris: José Corti.

SGARD, Jean (1975). "Antoine François Prévost". In: Histoire littéraire de la France (de 1715 à 1789), t. III. Paris: Les Editions Sociales, pp. 353-366.

SGARD, Jean (1984). L'Abbé Prévost. Labyrinthes de la mémoire. Paris: P.U.F.

SGARD, Jean (1995). Vingt études sur Prévost d'Exiles. Grenoble: Ellug. 